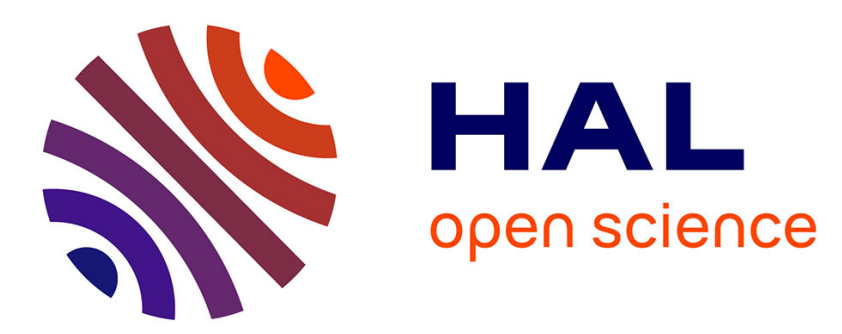

\title{
A Multiscale Computational Approach for Contact Problems
}

\author{
Pierre Ladevèze, Anthony Nouy, O. Loiseau
}

\section{To cite this version:}

Pierre Ladevèze, Anthony Nouy, O. Loiseau. A Multiscale Computational Approach for Contact Problems. Computer Methods in Applied Mechanics and Engineering, 2002, 191 (43), pp.4869-4891. 10.1016/S0045-7825(02)00406-1 . hal-00366657

\section{HAL Id: hal-00366657 https://hal.science/hal-00366657}

Submitted on 9 Mar 2009

HAL is a multi-disciplinary open access archive for the deposit and dissemination of scientific research documents, whether they are published or not. The documents may come from teaching and research institutions in France or abroad, or from public or private research centers.
L'archive ouverte pluridisciplinaire HAL, est destinée au dépôt et à la diffusion de documents scientifiques de niveau recherche, publiés ou non, émanant des établissements d'enseignement et de recherche français ou étrangers, des laboratoires publics ou privés. 


\title{
A Multiscale Computational Approach for Contact Problems
}

\author{
P. Ladeveze ${ }^{a}$ A. Nouy ${ }^{a}$ O. Loiseau ${ }^{a}$ \\ ${ }^{a}$ LMT-Cachan (ENS Cachan / CNRS / Université Paris VI) \\ 61 Avenue du Président Wilson, F-94235 Cachan CEDEX, France
}

\begin{abstract}
We introduce a two-scale computational strategy for the resolution of contact problems with friction, possibly with numerous contact surfaces; the structure studied may also be highly heterogeneous. The description of "micro" and "macro" quantities is performed on the interfaces arising from the decomposition of the structure studied into an assembly of substructures and interfaces. The efficiency and robustness of the method are illustrated on several examples. This iterative computational strategy is suitable for parallel computing; it can be interpreted as a mixed multilevel domain decomposition method. Its scalability has been demonstrated and comparisons with other domain decomposition methods are also given.
\end{abstract}

Key words: Contact, friction, multiscale, domain decomposition, LATIN method

\section{Introduction}

The numerical resolution of engineering contact problems is probably one of the most difficult and demanding tasks in computational structural mechanics, which explains why so many works have been devoted to contact problems. Without being exhaustive, let us mention [10,23], whose main feature consists of formulating the problem as an implicit variational inequality. There are many approaches to the resolution of the global problem, such as penalty or Lagrange multiplier methods [30,14,28,29]. The former [13] are based on the regularization of contact constraints and, usually, lead to ill-conditioned problems when dealing with large-scale systems. Among the latter are mathematical programming techniques [15] and gradient methods $[21,7,25,26]$. One can also mention methods which use the augmented Lagrangian formulation or mixed approaches $[27,15]$ or mixed approaches [1] close to an augmented Lagrangian formulation. 
However, the treatment of large-scale problems using global methods often gets expensive. Recently, domain decomposition methods have been introduced to accelerate the resolution of contact problems $[5,6,8,2,4]$. More recently, the requirement of scalability for this type of method has led to the extension to contact problems of the FETI method [9].

This paper introduces an alternative computational method. The key point lies in the observation that what causes difficulties in contact problems is the fact that the local solution includes high gradient effects with a short wavelength compared to the characteristic length of the structure studied. This is very clear, for example, for cracks involving contact with friction: at the crack's tip, stress singularities occur. It follows that contact problems, just like problems involving highly heterogeneous structures (such as composite structures), belong to the class of problems in which phenomena with different wavelengths interact. Consequently, the proposed computational strategy for contact problems is an extension of the micro-macro computational strategy developed for highly heterogeneous structures [18-20], which incorporates a homogenization procedure. This last computational strategy is also an enhancement of a previous one $[5,6]$.

The first step consists of decomposing the structure into an assembly of simple constituents, i.e. substructures and interfaces. For instance, a substructure may contain one or several cells of a composite structure. Each of these components possesses its own variables and equations. An interface transfers both a distribution of displacements and a distribution of forces.

Moreover, the unknowns (displacements and forces) are split at the interfaces into:

$$
\mathbf{s}=\mathbf{s}^{M}+\mathbf{s}^{m}
$$

where $\mathbf{s}^{M}$ is the set of the macroscopic quantities and $\mathbf{s}^{m}$ the complementary set of "micro" quantities. Our descriptions are based on a "continuousmedium" point of view and we use a general method for homogenization and local reanalysis. Here, contrary to previous works, we develop the tractionbased description, in which the equilibrium in traction is enforced at the interfaces [20]. For contact interfaces, we introduce contact laws with friction.

The second step of this micro-macro strategy consists of using the so-called LATIN method on the problem to be solved expressed as an assembly of substructures and interfaces. The resulting micro-macro strategy converges for stable materials under standard assumptions. Here, in order to focus on the main concepts, this method will be described only for linear elasticity.

At each iteration, one has to solve a linear "macro" problem defined on the entire homogenized structure along with a family of linear "micro" problems, each associated with a single substructure. For linear problems, this strat- 
egy involves a numerical parameter which can be interpreted as an interface stiffness.

Several numerical examples illustrate the efficiency and the robustness of the new approach.

Globally, this scheme is well-suited to parallel-architecture computers. It can be viewed as a mixed multilevel domain decomposition method. Its scalability has been proved numerically. In this paper, we also give comparisons with other domain decomposition methods. Let us also mention that a third scale can be introduced by discretizing the "macro" linear problem.

\section{The reference problem}

In this section, we outline the reference problem and its reformulation in terms of an assembly of substructures and interfaces [17]. Then, we introduce the contact conditions by conferring a specific behavior on the interfaces.

\subsection{Formulation of the problem}

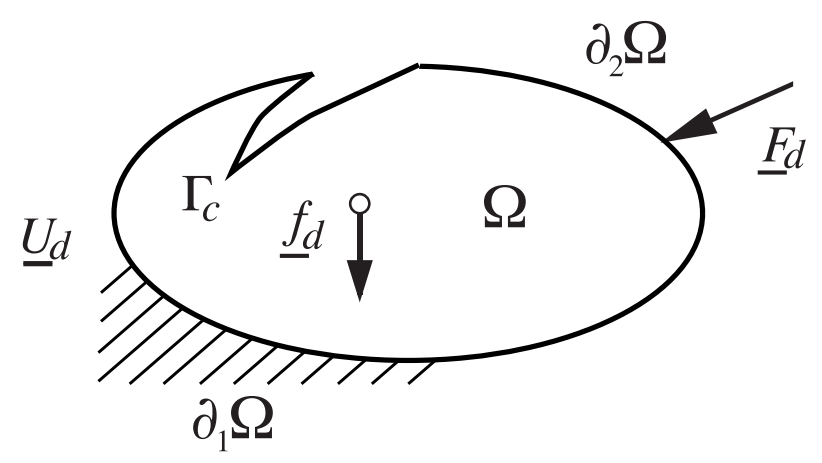

Fig. 1. The reference problem

We are considering the small perturbations of a structure $\Omega$ under prescribed loads and displacements defined by:

- a prescribed displacement $\underline{u}_{d}$ on part of the boundary $\partial_{1} \Omega$,

- a prescribed traction force $\underline{F}_{d}$ on part of the boundary $\partial_{2} \Omega$,

- a prescribed body force $\underline{f}_{d}$ on $\Omega$.

The structure has internal or external contact interfaces with friction designated by $\Gamma_{c}$. The reference problem will be presented in the case of linear elasticity. We designate the stress field by $\boldsymbol{\sigma}$ and the displacement field by $\underline{u}$, which belong respectively to finite energy spaces $\mathcal{S}$ and $\mathcal{U}$ defined on $\Omega$. 


\subsection{Reformulation of the problem}

The method we are using is not a classical domain decomposition method in that the body is split into two different types of entities: substructures and interfaces, both defined by their own variables and equations [17]. The structure is decomposed into a set $\mathbf{E}$ of non-overlapping substructures $\Omega_{E}$, which are subjected on their boundaries to a traction field $\underline{F}_{E}$ and to a displacement field $\underline{W}_{E}$. An interface $\Gamma_{E E^{\prime}}$ between two substructures $\Omega_{E}$ and $\Omega_{E^{\prime}}$ generates constitutive relations between $\left(\underline{W}_{E}, \underline{W}_{E^{\prime}}\right) \in \mathcal{W}_{E E^{\prime}}^{2}$ and $\left(\underline{F}_{E}, \underline{F}_{E^{\prime}}\right) \in \mathcal{F}_{E E^{\prime}}^{2}$ (see Figure 2) and allows us to take easily into account such complex behavior as contact with or without friction. Since both the displacement and the force at the interfaces are unknowns, the resulting approach is a "mixed" domain decomposition method as opposed to the primal and dual methods.
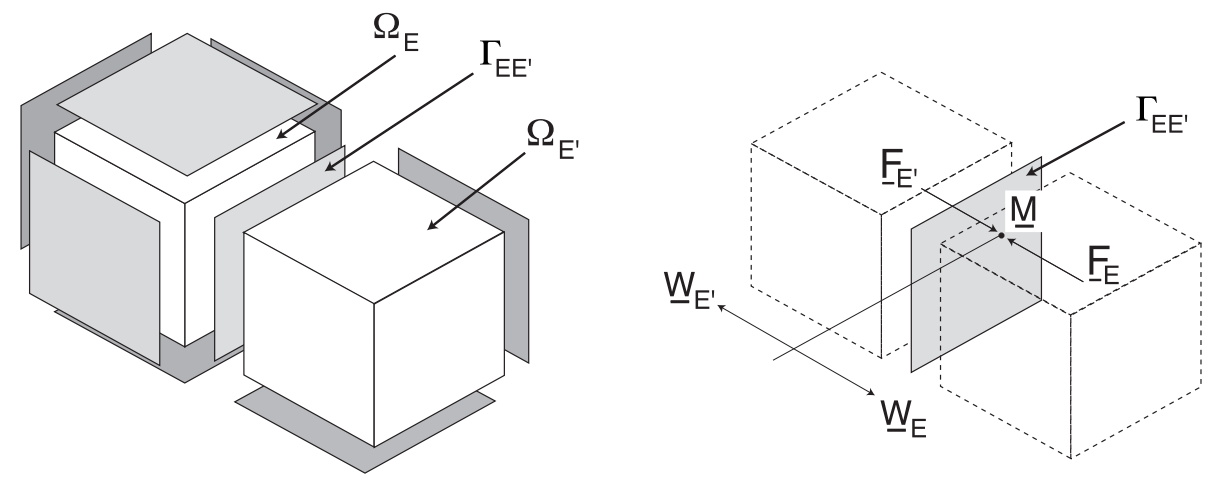

Fig. 2. Decomposition into substructures and interfaces

The problem can be rewritten as:

Find $\mathbf{s}=\left\{\mathbf{s}_{E} \mid E \in \mathbf{E}\right\}$ with $\mathbf{s}_{E}=\left(\underline{u}_{E}, \boldsymbol{\sigma}_{E}, \underline{W}_{E}, \underline{F}_{E}\right)$ which verify:

- a set of linear equations, possibly global in Space $\mathbf{A}_{d}$, such that, $\forall E \in \mathbf{E}$,

- Kinematic compatibility equations:

$$
\begin{aligned}
& \underline{u}_{E} \in \mathcal{U}_{E}, \quad \varepsilon_{E}=\varepsilon\left(\underline{u}_{E}\right), \\
& \underline{u}_{E \mid \partial \Omega_{E}}=\underline{W}_{E}
\end{aligned}
$$

- Equilibrium equations:

$$
\forall \underline{u}^{\star} \in \mathcal{U}_{E}, \int_{\Omega_{E}} \operatorname{Tr}\left[\boldsymbol{\sigma}_{E} \varepsilon\left(\underline{u}^{\star}\right)\right] d \Omega=\int_{\Omega_{E}} \underline{f}_{d} \cdot \underline{u}^{\star} d \Omega+\int_{\partial \Omega_{E}} \underline{F}_{E} \cdot \underline{u}_{\mid \partial \Omega_{E}}^{\star} d \Gamma
$$

- a set of equations $\boldsymbol{\Gamma}$, local in space and possibly nonlinear, such that, $\forall E \in$ $\mathrm{E}$, 
- Constitutive relation:

$$
\begin{aligned}
& \boldsymbol{\sigma}_{E}=\mathbf{K} \boldsymbol{\varepsilon}_{E} \text { on } \Omega_{E} \\
& \text { where } \mathbf{K} \text { is Hooke's tensor }
\end{aligned}
$$

- Interface behavior: $\forall E^{\prime} \in \mathbf{E}_{E}$,

$$
\left\{\begin{array}{l}
\underline{F}_{E}+\underline{F}_{E^{\prime}}=0 \text { (equilibrium) } \\
\underline{F}_{E}=\mathcal{A}_{\Gamma_{E E^{\prime}}}\left(\underline{W}_{E}, \underline{W}_{E^{\prime}}\right) \text { (constitutive relation) }
\end{array} \text { on } \Gamma_{E E^{\prime}}\right.
$$

where $\mathcal{A}_{\Gamma_{E E^{\prime}}}$ is the operator describing the interface's behavior.

where $\mathbf{E}_{E}$ is the set of subdomains adjacent to $E$, including interfaces with prescribed traction or displacement or external contact. This approach allows us to consider different types of interface behavior easily, e.g. perfect linkage or unilateral contact with or without friction. The set of equations $\mathbf{A}_{d}$ is solved without taking into account the set $\boldsymbol{\Gamma}$ and, consequently, it remains a quadratic minimization problem without constraints. Let us look at some examples of contact behavior.

\subsection{Examples of interface behavior}

All following equations are local at each point of an interface $\Gamma_{E E^{\prime}}$, for $E \in \mathbf{E}$ and $E^{\prime} \in \mathbf{E}_{E}$.

\subsubsection{Perfect linkage}

The linkage is defined by both the displacement and the traction force being continuous across the interface:

$$
\begin{aligned}
& \frac{\text { Perfect linkage }}{\underline{F}_{E}+\underline{F}_{E^{\prime}}=0} \\
& \underline{W}_{E}=\underline{W}_{E^{\prime}}
\end{aligned}
$$

\subsubsection{Unilateral contact without friction}

Following the formulation of static contact problems found in [10], let us use the resulting contact conditions. We designate by $\underline{n}$ the unit vector normal to $\Gamma_{E E^{\prime}}$ pointing from $\mathrm{E}$ to $\mathrm{E}^{\prime}$ and by $\mathbf{P}$ the corresponding orthogonal projection 
operator. The linkage is defined by:

$$
\begin{aligned}
& \text { Unilateral contact without friction } \\
& \underline{F}_{E}+\underline{F}_{E^{\prime}}=0 \\
& \underline{n} \cdot\left(\underline{W}_{E^{\prime}}-\underline{W}_{E}\right) \geqslant 0 \text { and } \underline{n} \cdot \underline{F}_{E} \leqslant 0 \\
& \left(\underline{n} \cdot\left(\underline{W}_{E^{\prime}}-\underline{W}_{E}\right)\right)\left(\underline{n} \cdot \underline{F}_{E}\right)=0 \\
& \mathbf{P} \underline{F}_{E}=\mathbf{P} \underline{F}_{E^{\prime}}=0
\end{aligned}
$$

\subsubsection{Unilateral contact with friction}

Here, the behavior is characterized by Coulomb's friction coefficient $f$. The notations are the same as before. The linkage is defined on $\Gamma_{E E^{\prime}}$ by:

\section{Unilateral contact with friction}

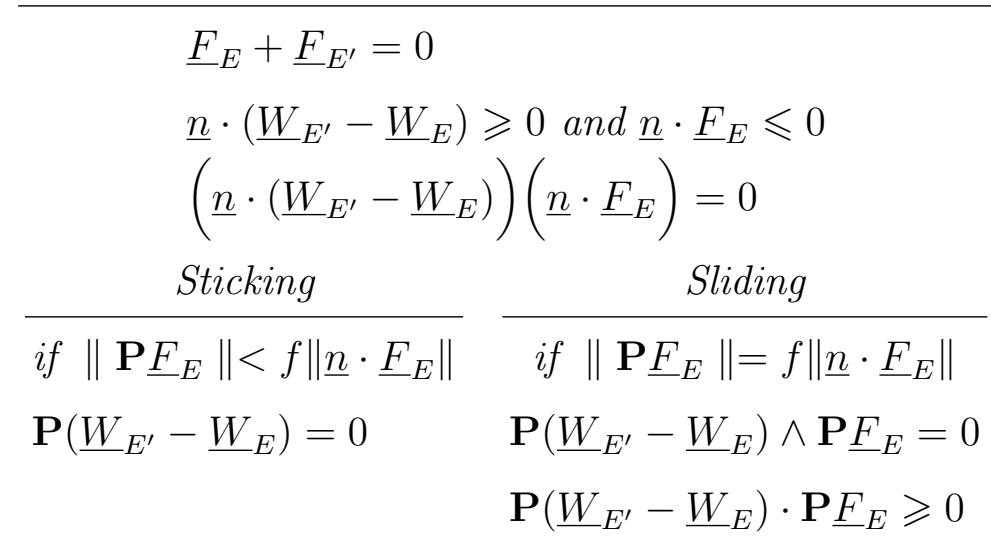

\section{Description of the micro-macro strategy}

The set of state variables is expected to have two parts: one, denoted M, related to the macroscale and the other, denoted $\mathrm{m}$, related to the microscale. Splitting between the micro and macro quantities is carried out only at the interfaces. The macro part is chosen to represent the large-wavelength solution. At every interface $\Gamma_{E E^{\prime}}$ such that $E \in \mathbf{E}$ and $E^{\prime} \in \mathbf{E}_{E}$, the interaction forces (or "interforces") and displacements are split into:

$$
\begin{aligned}
\underline{F}_{E \mid \Gamma_{E E^{\prime}}} & =\underline{F}_{E \mid \Gamma_{E E^{\prime}}}^{M}+\underline{F}_{E \mid \Gamma_{E E^{\prime}}}^{m} \\
\underline{W}_{E \mid \Gamma_{E E^{\prime}}} & =\underline{W}_{E \mid \Gamma_{E E^{\prime}}}^{M}+\underline{W}_{E \mid \Gamma_{E E^{\prime}}}^{m}
\end{aligned}
$$


Here, we are following the traction-based approach [20], which will be explained later. Splitting is performed using a finite-dimension projection operator $\Pi_{\mid \Gamma_{E E^{\prime}}}^{F}$ such that:

$$
\begin{gathered}
\underline{F}_{E \mid \Gamma_{E E^{\prime}}}^{M}=\boldsymbol{\Pi}_{\mid \Gamma_{E E^{\prime}}}^{F}\left(\underline{F}_{E \mid \Gamma_{E E^{\prime}}}\right) \\
\underline{F}_{E \mid \Gamma_{E E^{\prime}}}^{m}=\left(\mathbf{I}_{d}-\boldsymbol{\Pi}_{\mid \Gamma_{E E^{\prime}}}^{F}\left(\underline{F}_{E \mid \Gamma_{E E^{\prime}}}\right)\right.
\end{gathered}
$$

The corresponding macro part of the displacements comes from the duality on the contribution work:

$$
\begin{aligned}
& \left\langle\underline{F_{E}}, \underline{W_{E}}\right\rangle_{\Gamma_{E E^{\prime}}}=\left\langle\underline{F}_{E}^{m}, \underline{W}_{E}^{m}\right\rangle_{\Gamma_{E E^{\prime}}}+\left\langle\underline{F}_{E}^{M}, \underline{W}_{E}^{M}\right\rangle_{\Gamma_{E E^{\prime}}} \\
& \text { where }\left\langle\underline{F}_{E}, \underline{W}_{E}\right\rangle_{\Gamma_{E E^{\prime}}}=\int_{\Gamma_{E E^{\prime}}} \underline{F}_{E \mid \Gamma_{E E^{\prime}}} \cdot \underline{W_{E \mid \Gamma_{E E^{\prime}}}} d \Gamma
\end{aligned}
$$

For instance, the projector $\Pi_{\mid \Gamma_{E E^{\prime}}}^{F}$ can be chosen in order to extract the resultant and moment of Field $\underline{F}_{E}$ on $\Gamma_{E E^{\prime}}$. The new interface quantities are now:

$$
\begin{aligned}
\left(\underline{W}^{M}, \underline{F}^{M}\right) & \in \mathcal{W}_{a d}^{M} \times \mathcal{F}_{a d}^{M} \\
\left(\underline{W}^{m}, \underline{F}^{m}\right) & \in \mathcal{W}^{m} \times \mathcal{F}^{m}
\end{aligned}
$$

Following the traction-based strategy, we choose the space $\mathcal{F}_{\text {ad }}^{M}$ such that $\underline{F}^{M}$ achieves the transmission conditions at each interface and is in equilibrium with $\underline{f}_{d}$ in each substructure.

$$
\begin{aligned}
& \mathcal{F}_{a d}^{M}=\left\{\underline{F}^{M} \in \mathcal{F}^{M} / \forall E \in \mathbf{E}, \quad \forall E^{\prime} \in \mathbf{E}_{E}, \underline{F}_{E}^{M}+\underline{F}_{E^{\prime}}^{M}=0 \quad \text { on } \Gamma_{E E^{\prime}}\right. \\
& \left.\underline{F}_{E}^{M} \text { in equilibrium with } \underline{f}_{d}\right\}
\end{aligned}
$$

Let us note that these conditions are verified even in the case of unilateral contact. There is no restriction in the choice of the space $\mathcal{W}_{a d}^{M}=\mathcal{W}^{M}$.

\section{Computational micro-macro strategy}

The problem becomes: find the micro and macro displacement and interforce at the interface which verify both sets of equations $\mathbf{A}_{d}$ (kinematic and equilibrium equations on each substructure) and $\boldsymbol{\Gamma}$ (constitutive relation and interface's behavior). To solve this problem, we are using the framework of the LATIN method applied to the degenerated case where time has disappeared [17]. This procedure builds successively an element $\mathbf{s}$ of the space of admissible fields $\mathbf{A}_{d}$ and an element of the second set $\boldsymbol{\Gamma}$ at each iteration. Iteration $n$ starts with $\mathbf{s}_{n} \in \mathbf{A}_{d}$. Then, the local stage is performed from this element to an element 
$\widehat{\mathbf{s}}_{n+1 / 2} \in \boldsymbol{\Gamma}$ using the upward search direction $\mathbf{E}^{+}$. Next, the linear stage is performed using the downward search direction $\mathbf{E}^{-}$(see Figure 3), leading from $\widehat{\mathbf{s}}_{n+1 / 2}$ to $\mathbf{s}_{n+1} \in \mathbf{A}_{d}$. The search directions are the parameters of the method.

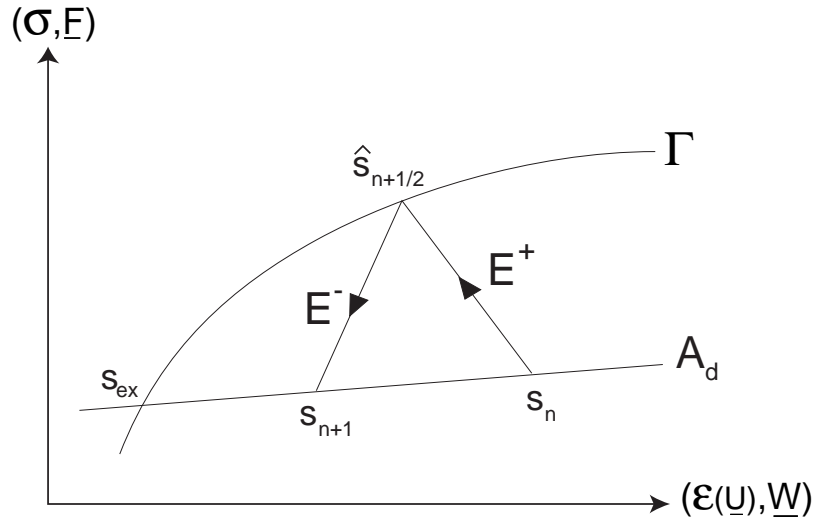

Fig. 3. An iteration of the LATIN method

\subsection{The local stage}

The problem can be written:

For a given $\mathbf{s}_{n} \in \mathbf{A}_{d}$, find $\widehat{\mathbf{s}}_{n+1 / 2} \in \boldsymbol{\Gamma}$ such that $\forall E \in \mathbf{E}$

- $\widehat{\boldsymbol{\sigma}}_{E, n+1 / 2}-\boldsymbol{\sigma}_{E, n}+\mathbf{K}\left(\widehat{\boldsymbol{\varepsilon}}_{E, n+1 / 2}-\boldsymbol{\varepsilon}_{E, n}\right)=0$

- $\forall E^{\prime} \in \mathbf{E}_{E}, \quad \forall\left(\underline{F}^{m^{*}}, \underline{F}^{M^{*}}\right) \in \mathcal{F}_{E E^{\prime}}^{m} \times \mathcal{F}_{E E^{\prime}}^{M}$,

$$
\begin{aligned}
& \left\langle\frac{1}{k_{m}}\left(\underline{\widehat{F}}_{E, n+1 / 2}^{m}-\underline{F}_{E, n}^{m}\right)-\left(\underline{\widehat{W}}_{E, n+1 / 2}^{m}-\underline{W}_{E, n}^{m}\right), \underline{F}^{m^{*}}\right\rangle_{\Gamma_{E E^{\prime}}}=0 \\
& \left\langle\frac{1}{k_{M}}\left(\underline{\widehat{F}}_{E, n+1 / 2}^{M}-\underline{F}_{E, n}^{M}\right)-\left(\underline{\widehat{W}}_{E, n+1 / 2}^{M}-\underline{W}_{E, n}^{M}\right), \underline{F}^{M^{*}}\right\rangle_{\Gamma_{E E^{\prime}}}=0
\end{aligned}
$$

where $k_{m}$ and $k_{M}$ are the parameters of the method. Note that the problem is formulated in such a way that no distinction is made between boundary interfaces and interfaces between subdomains; thus, the boundary conditions are taken into account easily and exactly. Let us note that for perfect linkage it has been proved that the optimum value of $k_{M}$ is $+\infty$ [20]. Therefore, here, we are taking a rather large value which, practically, is $k_{m}$.

\section{Example of unilateral contact with friction}

In the case of contact interfaces, taking the same parameter for the downward and upward search directions, the set of equations $\boldsymbol{\Gamma}$ remains local in space. At 
the beginning of this stage, the complete interforce $\underline{F}$ is assembled. Using the behavior (1) and the equations of the search direction (6) and (7), all possible cases (separation, contact with sliding or sticking) can be calculated easily at each point of the interface from the known element $\mathbf{s}_{n}$. To simplify the writing, let us omit the index $n+1 / 2$. To solve this stage, we use the procedure of Table (1).

Table 1

Solution of the local stage in the case of a contact interface with friction

\begin{tabular}{|c|c|}
\hline $\begin{array}{r}\text { First } \\
N_{n}=\frac{k}{2}\left(\underline{W}_{E^{\prime}, n}-\underline{W}_{E, n}\right) \\
T_{n}=\frac{k}{2} \mathbf{P}\left(\underline{W}_{E^{\prime}, n}-\underline{W}_{E},\right. \\
\mathbf{S e p}\end{array}$ & $\begin{array}{l}\text { lculation } \\
\underline{n}-\frac{1}{2 k}\left(\underline{F}_{E^{\prime}, n}-\underline{F}_{E, n}\right) \cdot \underline{n} \\
-\frac{1}{2} \mathbf{P}\left(\underline{F}_{E^{\prime}, n}-\underline{F}_{E, n}\right) \\
\text { ration }\end{array}$ \\
\hline $\begin{array}{l}\widehat{\widehat{F}}_{E}=\widehat{\widehat{F}}_{E} \\
\widehat{\widehat{W}}_{E}=\underline{W} \\
\widehat{\widehat{W}}_{E^{\prime}}=\underline{W}\end{array}$ & $\begin{array}{l}n>0 \\
=0 \\
n-\frac{1}{k} \underline{F}_{E, n} \\
{ }_{, n}-\frac{1}{k} \underline{F}_{E^{\prime}, n} \\
\text { thact }\end{array}$ \\
\hline $\begin{array}{l}\widehat{\widehat{F}}_{E} \cdot \underline{n}=-\widehat{\widehat{F}}_{E^{\prime}} \cdot \underline{n}=N_{n} \\
\widehat{\widehat{W}}_{E} \cdot \underline{n}=\frac{1}{2}\left(\left(\underline{W}_{E, n}+\underline{W}\right.\right. \\
\widehat{\widehat{W}}_{E^{\prime}}=\underline{W}_{E} \\
g_{n}=\end{array}$ & $\begin{array}{l}n \leqslant 0 \\
\left.\left.{ }_{, n}\right)-\frac{1}{k}\left(\underline{F}_{E, n}+\underline{F}_{E^{\prime}, n}\right)\right) \cdot \underline{n} \\
\widehat{\underline{F}}_{E, n} \cdot \underline{n}\end{array}$ \\
\hline Sticking & Sliding \\
\hline $\begin{array}{c}\text { if }\left\|T_{n}\right\|<g_{n} \\
\mathbf{P} \underline{\widehat{F}}_{E}=-\mathbf{P} \widehat{\widehat{F}}_{E^{\prime}}=T_{n} \\
\mathbf{P} \widehat{\widehat{W}}_{E}=\mathbf{P} \underline{W}_{E, n}+\frac{1}{k} \mathbf{P}\left(\underline{\widehat{F}}_{E}-\underline{F}_{E, n}\right) \\
\mathbf{P} \underline{\widehat{W}}_{E^{\prime}}=\mathbf{P} \underline{\widehat{W}}_{E}\end{array}$ & $\begin{array}{c}\text { if }\left\|T_{n}\right\| \geqslant g_{n} \\
\mathbf{P} \underline{\widehat{F}}_{E}=-\mathbf{P} \underline{\widehat{F}}_{E^{\prime}}=g_{n} \frac{T_{n}}{\left\|T_{n}\right\|} \\
\mathbf{P} \underline{\widehat{W}}_{E}=\mathbf{P} \underline{W}_{E, n}+\frac{1}{k} \mathbf{P}\left(\underline{\widehat{F}}_{E}-\underline{F}_{E, n}\right) \\
\mathbf{P} \underline{\widehat{W}}_{E^{\prime}}=\mathbf{P} \underline{W}_{E^{\prime}, n}+\frac{1}{k} \mathbf{P}\left(\underline{\widehat{F}}_{E^{\prime}}-\underline{F}_{E^{\prime}, n}\right)\end{array}$ \\
\hline
\end{tabular}

\subsection{The linear stage}

The linear stage can be written: 
For a given $\widehat{\mathbf{s}}_{n+1 / 2} \in \boldsymbol{\Gamma}$, find $\mathbf{s}_{n+1} \in \mathbf{A}_{d}$ such that

- $\forall E \in \mathbf{E}, \quad \boldsymbol{\sigma}_{E, n+1}-\widehat{\boldsymbol{\sigma}}_{E, n+1 / 2}-\mathbf{K}\left(\boldsymbol{\varepsilon}_{E, n+1}-\widehat{\boldsymbol{\varepsilon}}_{E, n+1 / 2}\right)=0$

- $\forall E \in \mathbf{E}, \quad \forall E^{\prime} \in \mathbf{E}_{E}, \quad \forall \underline{F}^{m^{*}} \in \mathcal{F}_{E E^{\prime}}^{m}$,

$\left\langle\frac{1}{k_{m}}\left(\underline{F}_{E, n+1}^{m}-\underline{\widehat{F}}_{E, n+1 / 2}^{m}\right)+\left(\underline{W}_{E, n+1}^{m}-\underline{\widehat{W}}_{E, n+1 / 2}^{m}\right), \underline{F}^{m^{*}}\right\rangle_{\Gamma_{E E^{\prime}}}=0$

- $\forall \underline{F}^{M^{*}} \in \mathcal{F}_{a d, 0}^{M}$,

$$
\begin{aligned}
\sum_{E \in \mathbf{E}} \sum_{E^{\prime} \in \mathbf{E}_{E}}\langle & \frac{1}{k_{M}}\left(\underline{F}_{E, n+1}^{M}-\underline{\widehat{F}}_{E, n+1 / 2}^{M}\right)+ \\
& \left.\left(\underline{W}_{E, n+1}^{M}-\underline{\widehat{W}}_{E, n+1 / 2}^{M}\right), \underline{F}^{M^{*}}\right\rangle_{\Gamma_{E E^{\prime}}}=0
\end{aligned}
$$

We can see that the choice of the admissible space $\mathcal{F}_{\text {ad }}^{M}$ makes the macro search direction global. Omitting the index $n$, finding $\mathbf{s} \in \mathbf{A}_{d}$ can be written:

Find $\mathbf{s}=\left\{\mathbf{s}_{E} \mid E \in \mathbf{E}\right\}$

with $\mathbf{s}_{E}=\left(\underline{u}_{E}, \boldsymbol{\sigma}_{E}, \underline{W}_{E}^{m}, \underline{W}_{E}^{M}, \underline{F}_{E}^{m}, \underline{F}_{E}^{M}\right)$ which verifies :

- Kinematic admissibility equations:

$$
\begin{aligned}
& \underline{W}^{m} \in \mathcal{W}^{m}, \underline{W}^{M} \in \mathcal{W}_{a d}^{M}, \forall E \in \mathbf{E}, \underline{u}_{E} \in \mathcal{U}_{E}, \\
& \forall E^{\prime} \in \mathbf{E}_{E}, \underline{u}_{E \mid \Gamma_{E E^{\prime}}}=\underline{W}_{E}^{m}+\underline{W}_{E}^{M}
\end{aligned}
$$

- Equilibrium equations:

$$
\begin{gathered}
\underline{F}^{m} \in \mathcal{F}^{m}, \quad \underline{F}^{M} \in \mathcal{F}_{a d}^{M}, \quad \forall E \in \mathbf{E}, \quad \boldsymbol{\sigma}_{E} \in \mathcal{S}_{E}, \quad \forall \underline{u}^{\star} \in \mathcal{U}_{E} \\
\int_{\Omega_{E}} \operatorname{Tr}\left[\boldsymbol{\sigma}_{E} \boldsymbol{\varepsilon}\left(\underline{u}^{\star}\right)\right] d \Omega=\int_{\Omega_{E}} \underline{f}_{d} \cdot \underline{u}^{\star} d \Omega \quad+ \\
\int_{\partial \Omega_{E}} \underline{F}_{E}^{M} \cdot \underline{u}_{\mid \partial \Omega_{E}}^{\star} d \Gamma+\int_{\partial \Omega_{E}} \underline{F}_{E}^{m} \cdot \underline{u}_{\mid \partial \Omega_{E}}^{\star} d \Gamma
\end{gathered}
$$

The resulting problem is defined on the whole structure; it is characterized a priori by highly coupled macro and micro unknowns. However, we will see that this coupling can be taken into account by a homogenization procedure. The characteristic length of the substructure defines the microscale; the macroscale is associated with the characteristic length of the whole structure. In the traction-based strategy, we recall that $\underline{F}^{M} \in \mathcal{F}_{a d}^{M}$ contains the subspace of the interforces which verify the equilibrium conditions at the interfaces, even in the case of contact interfaces. 


\subsubsection{The micro problem}

Because of the search directions (8) and (9) for micro quantities, the local problem in Substructure $E$ can be rewritten:

$$
\begin{aligned}
& \text { Find } \underline{u}_{E} \in \mathcal{U}_{E} \text { which verifies } \forall \underline{u}^{\star} \in \mathcal{U}_{E} \\
& \int_{\Omega_{E}} \operatorname{Tr}\left[\mathbf{K} \varepsilon\left(\underline{u}_{E}\right) \varepsilon\left(\underline{u}^{\star}\right)\right] d \Omega+\int_{\partial \Omega_{E}} k\left(\mathbf{I}_{d}-\boldsymbol{\Pi}^{U}\right) \underline{u}_{E \mid \partial \Omega_{E}} \cdot\left(\mathbf{I}_{d}-\mathbf{\Pi}^{U}\right) \underline{u}_{\mid \partial \Omega_{E}}^{\star} d \Gamma= \\
& \int_{\Omega_{E}} \underline{f}_{d} \cdot \underline{u}^{\star} d \Omega+\int_{\partial \Omega_{E}} \underline{F}_{E}^{M} \cdot \underline{u}_{\mid \partial \Omega_{E}}^{\star} d \Gamma+\int_{\partial \Omega_{E}}\left(\underline{\widehat{F}}_{E}^{m}+k \underline{W}_{E}^{m}\right) \cdot \underline{u}_{\mid \partial \Omega_{E}}^{\star} d \Gamma
\end{aligned}
$$

where $\boldsymbol{\Pi}^{U}$ is the macro projector for the displacement arising from the duality on the contribution work. The choice of a macro projector $\boldsymbol{\Pi}^{F}$ which preserves the resultant and the moment implies that the rigid body modes are the kernel of the bilinear form on the left-hand side. Consequently, this problem has a solution with an undefined additional rigid body mode displacement field if $\underline{F}_{E}^{M}$ is in equilibrium with $\underline{f}_{d}$. We note that this condition is fulfilled by the choice of the approximation space $\mathcal{F}_{a d}^{M}$.

Since the micro problem is linear, its right-hand side can be separated into a contribution $\left(\underline{f}_{d}, \widehat{\underline{F}}_{E}^{m}+k \underline{\underline{W}}_{E}^{m}\right)$ on the microscale and a contribution $\underline{F}^{M}$ on the macroscale. Therefore, the solution to the microscale problem (13) and, in particular, the macro part of the boundary displacement can be written:

$$
\underline{W}_{E}^{M}=\boldsymbol{\Pi}^{U} \underline{u}_{\mid \partial \Omega_{E}}=\widehat{W}_{d}^{M}\left(\underline{f}_{d}, \widehat{\widehat{F}}_{E}^{m}+k \underline{\widehat{W}}_{E}^{m}\right)+\mathbf{L}_{E}\left(\underline{F}_{E}^{M}\right)+\boldsymbol{\Pi}^{U} \underline{\alpha}_{E \mid \partial \Omega_{E}}
$$

where $\widehat{W}_{d}^{M}$ is the macro contribution of the micro right-hand side and $\underline{\alpha}_{E}$ is an additional rigid body mode. $\mathbf{L}_{E}: \mathcal{F}_{a d}^{M} \longrightarrow \mathcal{W}^{M}$ is a finite-dimension operator which can be interpreted as a homogenized behavior operator for Cell $E$; it is defined, for example, by prescribing zero values for the resultant and the moment of the displacement on the edge of E. $\mathbf{L}_{E}$ represents a symmetric, positive definite bilinear form on $\mathcal{F}_{a d, 0}^{M} \times \mathcal{F}_{a d, 0}^{M} . \mathbf{L}_{E}$ can be calculated by solving a small number of problems on the microscale because, in the cell, $\underline{F}_{M}$ depends on only few parameters.

\subsubsection{The macro problem}

The macro problem consists of verifying the global macro search direction by taking into account the results obtained on the microscale (14). 


$$
\begin{aligned}
& \text { Find } \underline{F}^{M} \in \mathcal{F}_{a d}^{M} \text { such that, } \forall \underline{F}^{M^{*}} \in \mathcal{F}_{a d, 0}^{M}, \\
& \begin{aligned}
\sum_{E \in \mathbf{E}} \sum_{E^{\prime} \in \mathbf{E}_{E}}\left\langle\mathbf{L}_{E}\left(\underline{F}_{E}^{M}\right), \underline{F}^{M^{*}}\right\rangle_{\Gamma_{E E^{\prime}}}+\left\langle\frac{1}{k_{M}} \underline{F}_{E}^{M}, \underline{F}^{M^{*}}\right\rangle_{\Gamma_{E E^{\prime}}}= \\
\qquad \sum_{E \in \mathbf{E}} \sum_{E^{\prime} \in \mathbf{E}_{E}}\left\langle\frac{1}{k_{M}} \widehat{\widehat{F}}_{E}^{M}+\widehat{W}_{E}^{M}-\widehat{W}_{d}^{M}, \underline{F}^{M^{*}}\right\rangle_{\Gamma_{E E^{\prime}}}
\end{aligned}
\end{aligned}
$$

Due to the properties of Operator $\mathbf{L}_{E}$, the macro problem has a unique solution if $k \geq 0$. To guarantee the admissibility of $\underline{F}^{M}$, we can introduce Lagrange multipliers which are the rigid body modes of the substructures. We denote $\boldsymbol{\mathcal { R }}_{E}$ the space of the rigid body modes of Substructure $E$ and $\widetilde{\mathcal{F}}_{a d}^{M}$ the space of the macro interforces which verify the transmission conditions but not the equilibrium with $\underline{f}_{d}$ :

$$
\widetilde{\mathcal{F}}_{a d}^{M}=\left\{\underline{F}^{M} \in \mathcal{F}^{M} / \forall E \in \mathbf{E}, \forall E^{\prime} \in \mathbf{E}_{E}, \underline{F}_{E}^{M}+\underline{F}_{E^{\prime}}^{M}=0 \quad \text { on } \Gamma_{E E^{\prime}}\right\}
$$

The problem can be rewritten:

Find $\underline{F}^{M} \in \widetilde{\mathcal{F}}_{a d}^{M}$ and $\quad \underline{\alpha}=\left\{\underline{\alpha}_{E} \in \mathcal{R}_{E} \mid E \in \mathbf{E}\right\}$ such that:

- $\forall \underline{F}^{M^{*}} \in \widetilde{\mathcal{F}}_{a d, 0}^{M}$

$$
\begin{gathered}
\sum_{E \in \mathbf{E}} \sum_{E^{\prime} \in \mathbf{E}_{E}}\left\langle\mathbf{L}_{E}\left(\underline{F}_{E}^{M}\right), \underline{F}^{M^{*}}\right\rangle_{\Gamma_{E E^{\prime}}}+\left\langle\frac{1}{k_{M}} \underline{F}_{E}^{M}, \underline{F}^{M^{*}}\right\rangle_{\Gamma_{E E^{\prime}}}+\left\langle\underline{\alpha}_{E}, \underline{F}^{M^{*}}\right\rangle_{\Gamma_{E E^{\prime}}}= \\
\sum_{E \in \mathbf{E}} \sum_{E^{\prime} \in \mathbf{E}_{E}}\left\langle\frac{1}{k_{M}} \underline{\widehat{F}}_{E}^{M}+\widehat{W}_{E}^{M}-\widehat{W}_{d}^{M}, \underline{F}^{M^{*}}\right\rangle_{\Gamma_{E E^{\prime}}}
\end{gathered}
$$

- $\forall E, \forall \underline{\alpha}_{E}^{\star} \in \boldsymbol{\mathcal { R }}_{E}$

$$
\int_{\partial \Omega_{E}} \underline{F}_{E}^{M} \cdot \underline{\alpha}_{E \mid \partial \Omega_{E}}^{\star} d \Gamma+\int_{\Omega_{E}} \underline{f}_{d} \cdot \underline{\alpha}_{E}^{\star} d \Omega=0
$$

The resolution of the macro problem leads to the macro force field $\underline{F}^{M}$ and the additional rigid body modes $\underline{\alpha}_{E}$. Due to (13), we know the displacement solution in each substructure $E$. We can deduce its micro projection on the boundary $\underline{W}^{m}$ and we calculate, with respect to the micro search direction (9), the micro interforce $\underline{F}^{m}$. This completes the linear stage.

\subsection{Convergence results}

We are following the proof given in [17] for the monoscale approach. In the case of contact conditions, the micro and macro search parameters are chosen equal and positive. We use for $\mathbf{s}$ the following norm: 


$$
\begin{array}{r}
\|\mathbf{s}\|=\sum_{E \in \mathbf{E}} \int_{\Omega_{E}}\left\{\operatorname{Tr}\left[\boldsymbol{\sigma}_{E} \mathbf{K}^{-1} \boldsymbol{\sigma}_{E}\right]+\operatorname{Tr}\left[\boldsymbol{\varepsilon}\left(\underline{u}^{\star}\right) \mathbf{K} \varepsilon\left(\underline{u}^{\star}\right)\right]\right\} d \Omega+ \\
\sum_{E \in \mathbf{E}} \sum_{E^{\prime} \in \mathbf{E}_{E}} \int_{\Gamma_{E E^{\prime}}}\left\{\underline{F}_{E}^{m} \cdot k^{-1} \underline{F}_{E}^{m}+\underline{W}_{E}^{m} \cdot k \underline{W}_{E}^{m}+\right. \\
\left.\underline{F}_{E}^{M} \cdot k^{-1} \underline{F}_{E}^{M}+\underline{W}_{E}^{M} \cdot k \underline{W}_{E}^{M}\right\} d \Gamma
\end{array}
$$

In the case of contact without friction, we can prove that the mean value from two consecutive iterations converges towards the exact solution if $0<k_{1} \leqslant$ $k \leqslant k_{2}<\infty$. To guarantee convergence of the solution itself, we modify the procedure by introducing a relaxation after the linear stage $n+1$ and replacing the solution $\mathbf{s}_{n+1}$ by:

$$
\mathbf{s}_{n+1}-\mu\left(\mathbf{s}_{n+1}-\mathbf{s}_{n}\right) \longrightarrow \mathbf{s}_{n+1}
$$

The new procedure has been proved to be convergent for any positive $\mu$. In practice, we take $\mu=0.8$

\section{About discretization}

In this section, we present a consistent choice for the finite element approximation spaces.

First, let us consider the discretization of the interforce and of the displacement at the interface. A priori, the interforce density $\underline{F}_{E \mid \Gamma_{E E^{\prime}}}$ belongs to $H^{-1 / 2}\left(\Gamma_{E E^{\prime}}\right)$ and, therefore, is not necessarily continuous. Here, we make a natural choice by taking for $\mathcal{F}_{E, h}$ and $\widehat{\mathcal{F}}_{E, h}$ a space of piecewise continuous functions. The interface displacement appears only as an "interdisplacement" $\left(\widehat{W}_{E}-\widehat{W}_{E^{\prime}}\right)_{\mid \Gamma_{E E^{\prime}}}$ which can be considered as an interface deformation. Consequently, we can take for $\widehat{\underline{W}}_{E \mid \Gamma_{E E^{\prime}}}$ the same approximation space as for $\widehat{\underline{F}}_{E \mid \Gamma_{E E^{\prime}}}$.

$$
\mathcal{F}_{E, h}=\widehat{\mathcal{F}}_{E, h}=\widehat{\mathcal{W}}_{E, h}
$$

Therefore, the local stage is easy to define: the equations are simply written at the Gauss points and they remain local.

The last discretization choice concerns the resolution of the micro problem (13), for which we adopted a displacement approach. Then, the choice of the space approximation $\mathcal{U}_{E, h}$ for $\underline{u}_{E}$ is classical. We denote $\mathcal{W}_{E, h}$ the space approximation for $\underline{W}_{E}$ which is the trace on the boundary of $\mathcal{U}_{E, h}$ :

$$
\mathcal{W}_{E, h}=\left\{\underline{u}_{E \mid \partial \Omega_{E}} \mid \underline{u}_{E} \in \mathcal{U}_{E, h}\right\}
$$

In practice, if $\mathcal{F}_{E, h}$ is a space of piecewise continuous functions of degree $m$ $(m \geq 0)$, we could take for $\mathcal{U}_{E, h}$ a space of continuous functions of degree $p=$ 
$m+1$. Our experience shows that this classical discretization of the interforce and displacement generates spurious oscillating modes leading to numerical instability. This is due to the fact that the energy of several distributions of oscillating interforces is poorly calculated. This can be interpreted as a poor calculation of the local operator $\boldsymbol{A}_{E, h}$ such that

$$
\underline{u}_{E, h \mid \partial \Omega_{E}}=\boldsymbol{A}_{E, h}\left(\underline{F}_{E, h}\right)+\underline{W}_{d}
$$

To solve this problem, we propose to refine the discretization of Space $\mathcal{U}_{E}$ to have a better evaluation of the local operator $\boldsymbol{A}_{E, h}$. In practice, this can be done by refining the mesh near the boundary ("h-version") or by using a higher degree of approximation p for $\underline{u}_{h}$ near the boundary ("p-version").

We can see on Figure 4 the modification of the displacement approximation for the p- and h-versions.

INITIAL DISCRETIZATION
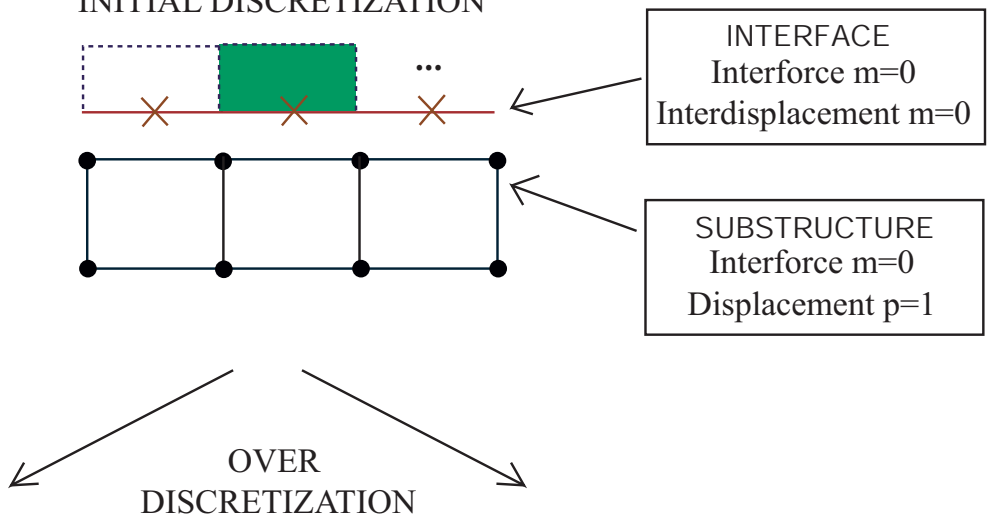

h-version

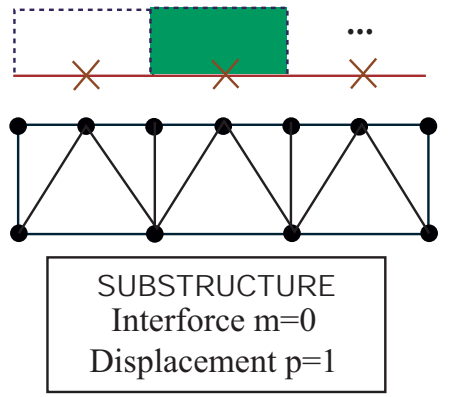

p-version

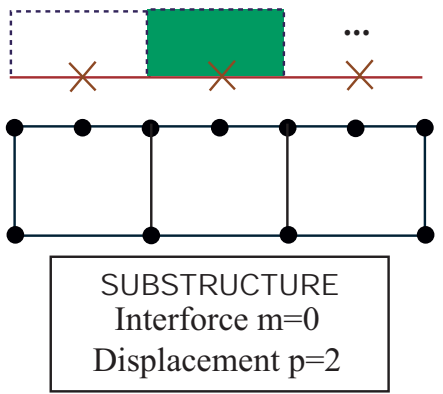

Fig. 4. Modification of the classical approximations of the interforce and local displacement for finite element calculations: h- and p-versions

This modification leads to a better solution without spurious modes and to a much higher convergence rate for the iterative strategy. Further details on the discretization will be given in an upcoming paper. 


\section{Examples}

In this section, we present two numerical tests which show the quality of the solution obtained and the efficiency of the algorithm. The multiscale method will be denoted MUSM, while the monoscale method will be denoted MOSM.

\subsection{Example 1: Square domain with contact boundary conditions}

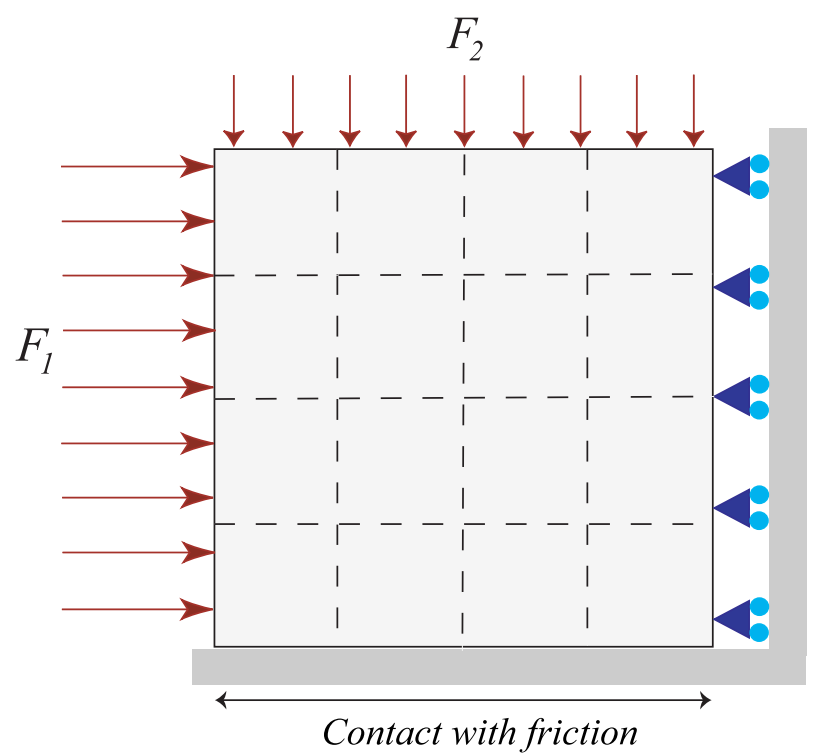

Fig. 5. The square domain with contact boundary conditions and its decomposition into subdomains

This example has been used by several laboratories. The reference solution can be found in [24]. It is very basic and has the advantage of providing different contact states. The problem consists of a square domain $\left(40 \times 40 \mathrm{~mm}^{2}\right)$ loaded by two interforce densities $F_{1}$ and $F_{2}$ on the left and upper sides respectively. There is a symmetry condition $\underline{u} \cdot x=0$ on the right-hand side and contact with friction on the lower side. We are assuming plane strain conditions with material coefficients $E=130000 \mathrm{~N} / \mathrm{mm}^{2}$ and $\nu=0.2$. In the reference above, the problem had been discretized with 33 nodes on the contact interface. To respect the overdiscretization of the local displacement in comparing our method with the reference problem, we chose QUA8 elements in the substructures for the displacements and piecewise constant functions for the interforces (32 equivalent points on the contact interface). This is equivalent to a "p-version" of overdiscretization with $p=2$. The structure was then decomposed into 16 square substructures which were discretized with $8 \times 8$ QUA8 elements. The macro spaces $\mathcal{W}^{M}$ and $\mathcal{F}^{M}$ were chosen to be equal and contain respectively the linear part of the displacement and the interforce at each interface, i.e.: 
- for the displacements: 2 translations, 1 rotation and 1 tensile strain per interface

- for the interforces: 2 resultants, 1 moment and 1 tensile force per interface

\section{Accuracy of the solution}

Now, let us compare the reference solution found in [24] with the solution using MUSM by looking in Table 2 at the length of the contact zones for different loading cases. Let us note that in MUSM the interforce and the interface displacement are considered to be piecewise constant. The mean values of these quantities lie at the midpoints of the interface elements. To measure a contact zone, we count the number of midpoints belonging to the zone and multiply this by the length of an element. We observe a perfect correspondence

Table 2

Length of the contact zones for different loading cases and Coulomb's friction coefficient: comparison between MUSM and the reference solution (REF)

\begin{tabular}{|c|c|c|cc|cc|cc|}
\hline$f$ & $\begin{array}{c}F_{1} \\
\left(\mathrm{~N} / \mathrm{mm}^{2}\right)\end{array}$ & $\begin{array}{c}F_{2} \\
\left(\mathrm{~N} / \mathrm{mm}^{2}\right)\end{array}$ & $\begin{array}{c}\text { Separate part } \\
(\mathrm{mm})\end{array}$ & $\begin{array}{c}\text { Sliding part } \\
(\mathrm{mm})\end{array}$ & $\begin{array}{c}\text { Sticking part } \\
(\mathrm{mm})\end{array}$ \\
\hline & & & $R E F$ & MUSM & REF & MUSM & REF & MUSM \\
\hline 1 & 100 & -50 & 3.75 & 3.75 & 18.75 & 20 & 17.5 & 16.25 \\
\hline 1 & 150 & -50 & 3.75 & 3.75 & 26.25 & 27.5 & 10 & 8.75 \\
\hline 0.2 & 100 & -50 & 0 & 0 & 40 & 40 & 0 & 0 \\
\hline 0.2 & 100 & -150 & 0 & 0 & 23.75 & 23.75 & 16.25 & 16.25 \\
\hline 0.2 & 100 & -250 & 0 & 0 & 3.75 & 3.75 & 36.25 & 36.25 \\
\hline
\end{tabular}

between the solution at convergence and the reference solution for all loading cases. We now focus on the first case of loading with $f=1$. We can also look at the distributions of the normal and tangential displacements on Figure 6 and of the normal and tangential generalized interforces on Figure 7 and observe that the solution at convergence is very close to the reference solution. The displacements and interforces of MUSM are considered at the midpoints.

\section{Convergence of the algorithm}

Figure 8 shows the evolution of the relative interface error (in $L_{2}$-norm) with the number of iterations. We note that only 10 iterations are sufficient to obtain a good approximation of the solution. We also note the improved convergence rate of MUSM compared with MOSM.

\section{$\square$ Influence of the discretization}

Figure 9 shows the evolution of the error with the number of iterations for 3 types of discretization: 


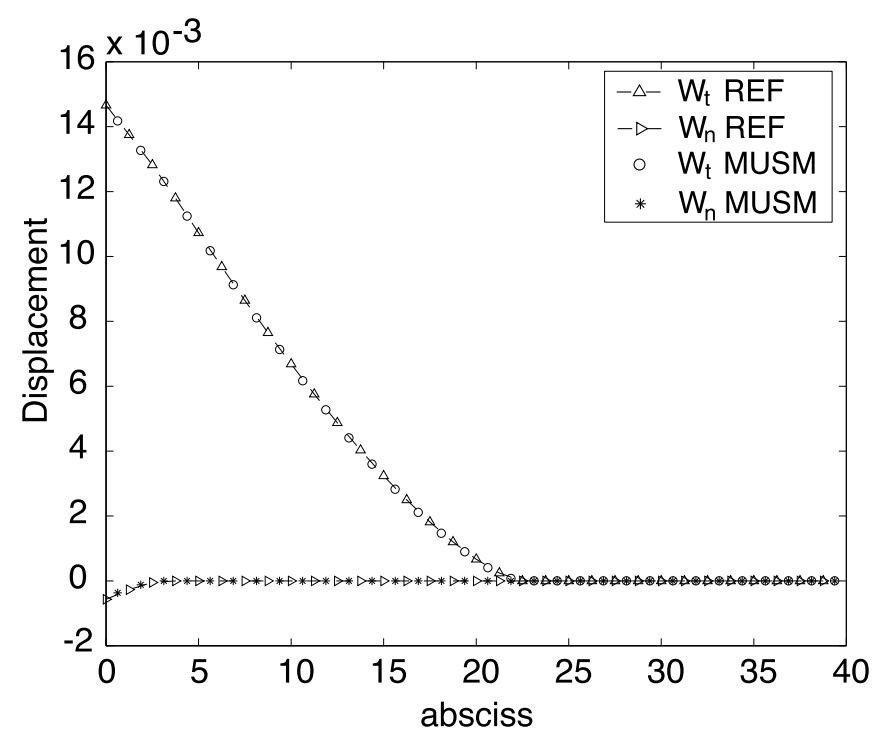

Fig. 6. Comparison with the reference solution: tangential and normal displacement at the contact interface

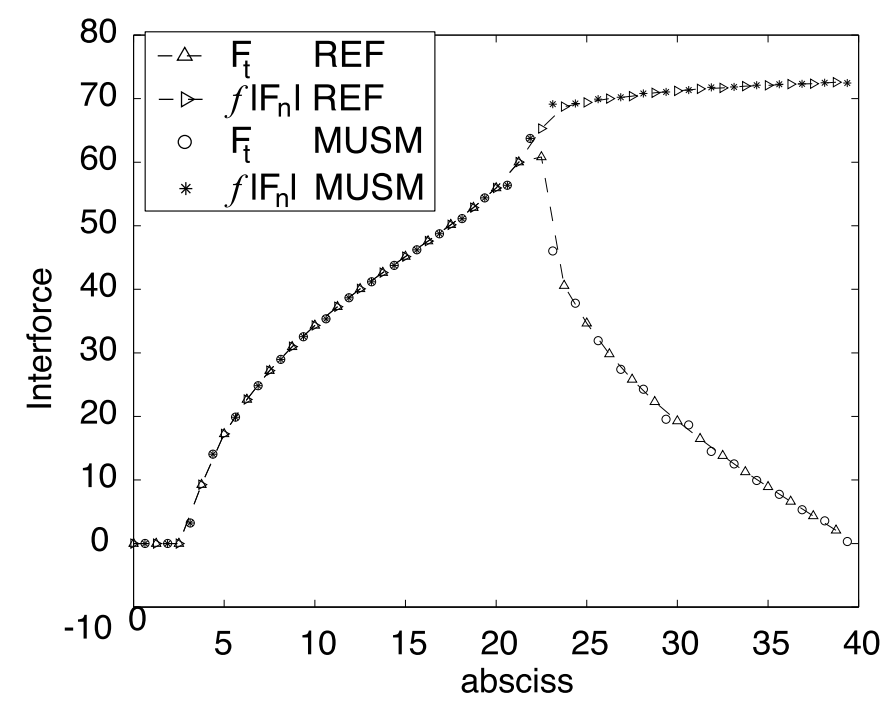

Fig. 7. Comparison with the reference solution: tangential and normal interforce at the contact interface

- $8 \times 8$ QUA4 for the displacement $(p=1)$ and linear approximation $\mathcal{P}_{1}$ for the interforce (dimension 9)

- $8 \times 8$ QUA4 for the displacement $(p=1)$ and piecewise constant $\mathcal{P}_{0}$ approximation for the interforce (dimension 8)

- $8 \times 8$ QUA8 for the displacement $(p=2)$ and piecewise constant $\mathcal{P}_{0}$ approximation for the interforce (dimension 8 ) $\longrightarrow$ overdiscretization.

Consequently, we conclude that the new type of discretization increases the convergence of the iterative strategy. 


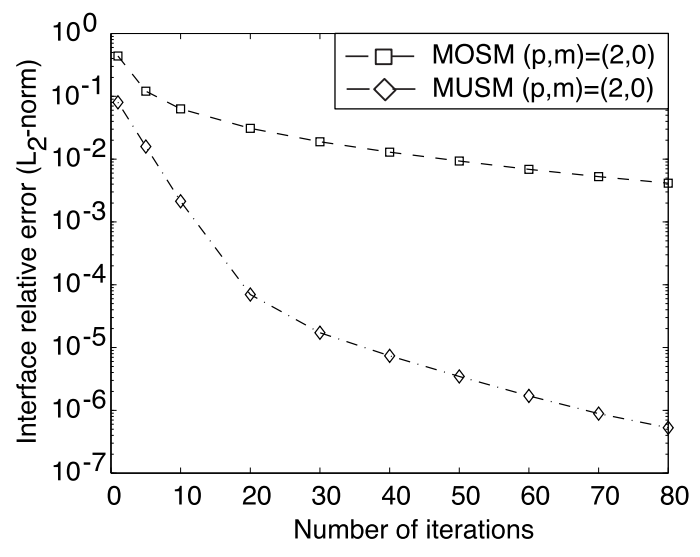

Fig. 8. Convergence of MOSM and MUSM

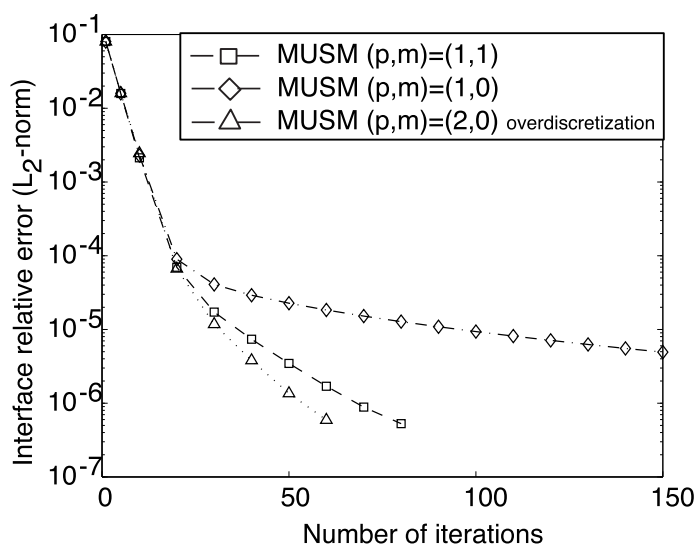

Fig. 9. Convergence of MUSM: influence of overdiscretization

\subsection{Example 2: Compression and bending of a domain with periodic cracks}

\subsubsection{Description of the 2D problem}

Here, we are attempting to solve a more complex problem. A square domain $\left(90 \times 90 \mathrm{~mm}^{2}\right)$ is loaded in compression $(100 \mathrm{Mpa})$ and bending (100 Mpa) along its upper side and fixed on its lower side (see Figure 10). The domain contains many cracks. We use contact interfaces to take into account the noninterpenetrating condition and the friction of the lips. We take $f=1$. We assume plane strain condition with material coefficients $E=130000 \mathrm{~N} / \mathrm{mm}^{2}$ and $\nu=0.2$. The domain decomposition and contact interfaces chosen are shown on Figure 10. Each substructure is discretized with $6 \times 6$ square QUA8 elements $(p=2)$. Each interface is discretized with $6 P_{0}$ elements $(m=0)$. This choice corresponds to a p-version of overdiscretization. The macro spaces $\mathcal{W}^{M}$ and $\mathcal{F}^{M}$ are chosen to be equal and contain respectively the linear part of the displacement and that of the interforce on each interface, as was the case for the previous example. 


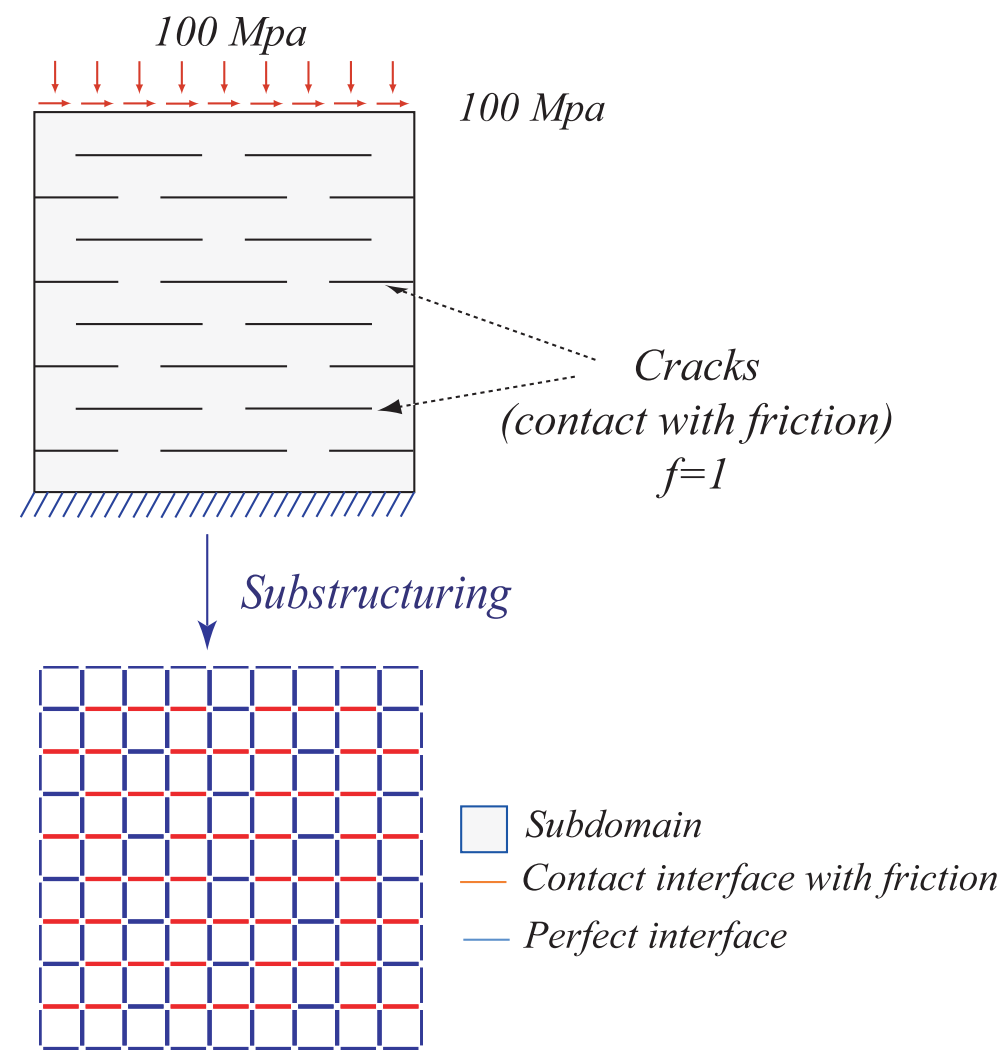

Fig. 10. The reference problem and the domain decomposition

\subsubsection{Solution given by the algorithm}

The solution in terms of Von Mises' stress and the interface displacement at convergence is shown on Figure 11. The leftward cracks are opening whereas

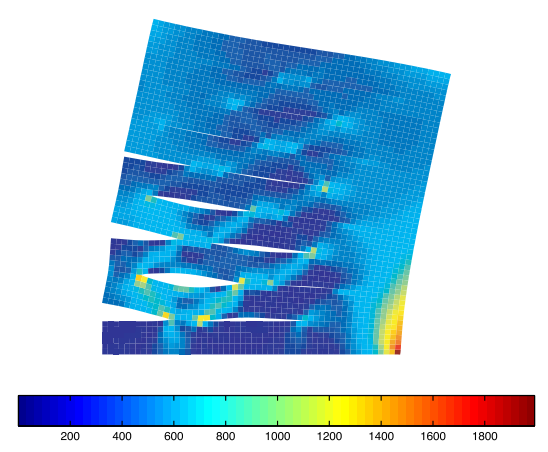

Mises Stress

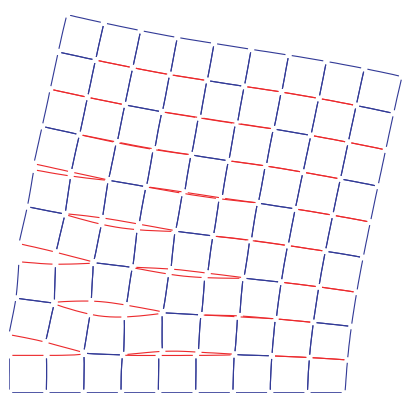

Interface displacement

Fig. 11. Solution at convergence: Von Mises' stress and interface displacement

the rightward cracks remain closed, sticking or sliding, as can be seen on Figure 12. On the same figure, we note that the solution to the macro problem $\underline{F}^{M}$ is a good approximation of the average solution. The micro term $\underline{F}^{m}$ improves the solution at the tips of the cracks. 


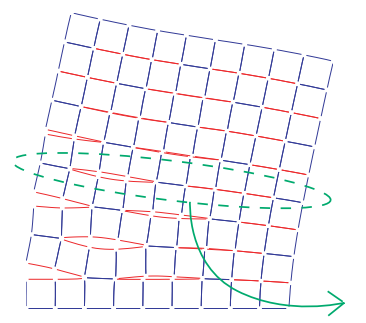

Interforce distribution

F
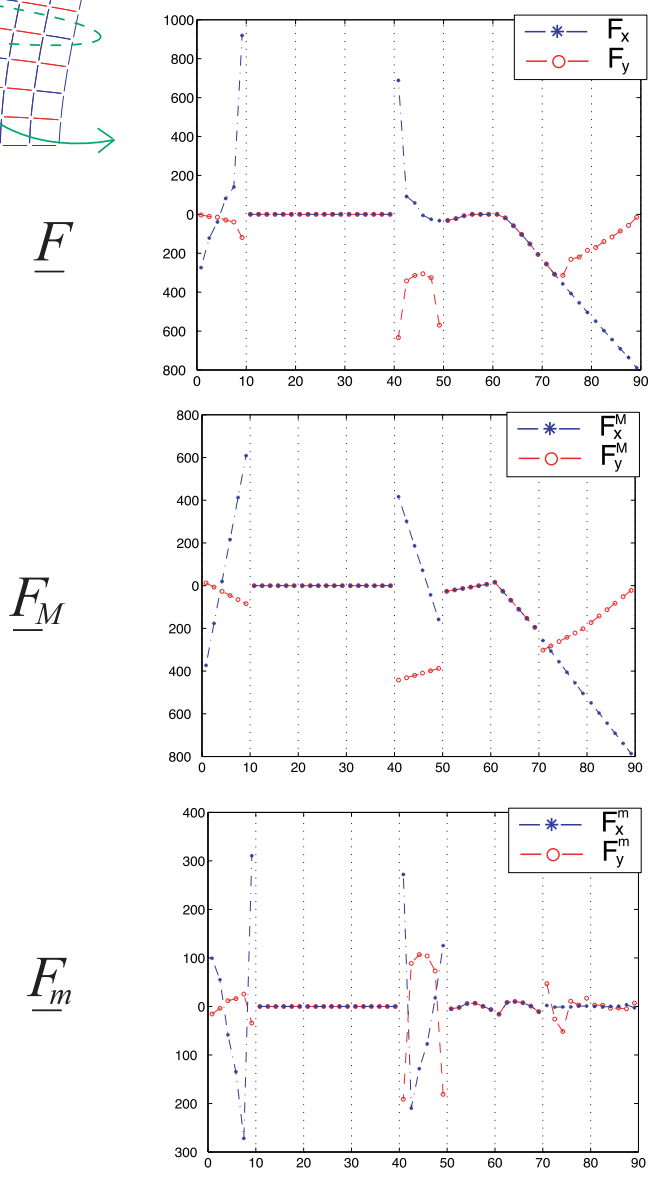

Fig. 12. Macro and micro interforces at convergence for some interfaces

\subsubsection{Evolution of the algorithm}

Now, let us look at the evolution of the solution with the number of iterations. Figures 13 and 14 show respectively the evolutions of $\underline{F}$ and of the corresponding contact error criterion on a sliding and sticking crack.

$$
\begin{array}{rll}
\text { Normal criterion } & :\left|\underline{F}_{E} \cdot \underline{n}\right| \cdot\left(\left(\underline{W}_{E^{\prime}}-\underline{W}_{E}\right) \cdot \underline{n}\right) \\
\text { Tangential criterion } & :\left(f\left|\underline{F}_{E} \cdot \underline{n}\right|-\left|\underline{F}_{E} \cdot \underline{t}\right|\right) \cdot\left|\left(\underline{W}_{E^{\prime}}-\underline{W}_{E}\right) \cdot \underline{t}\right|
\end{array}
$$

We conclude that the solution verifies the contact conditions well and converges rapidly. 


\section{Interforce $\mathrm{F}$}
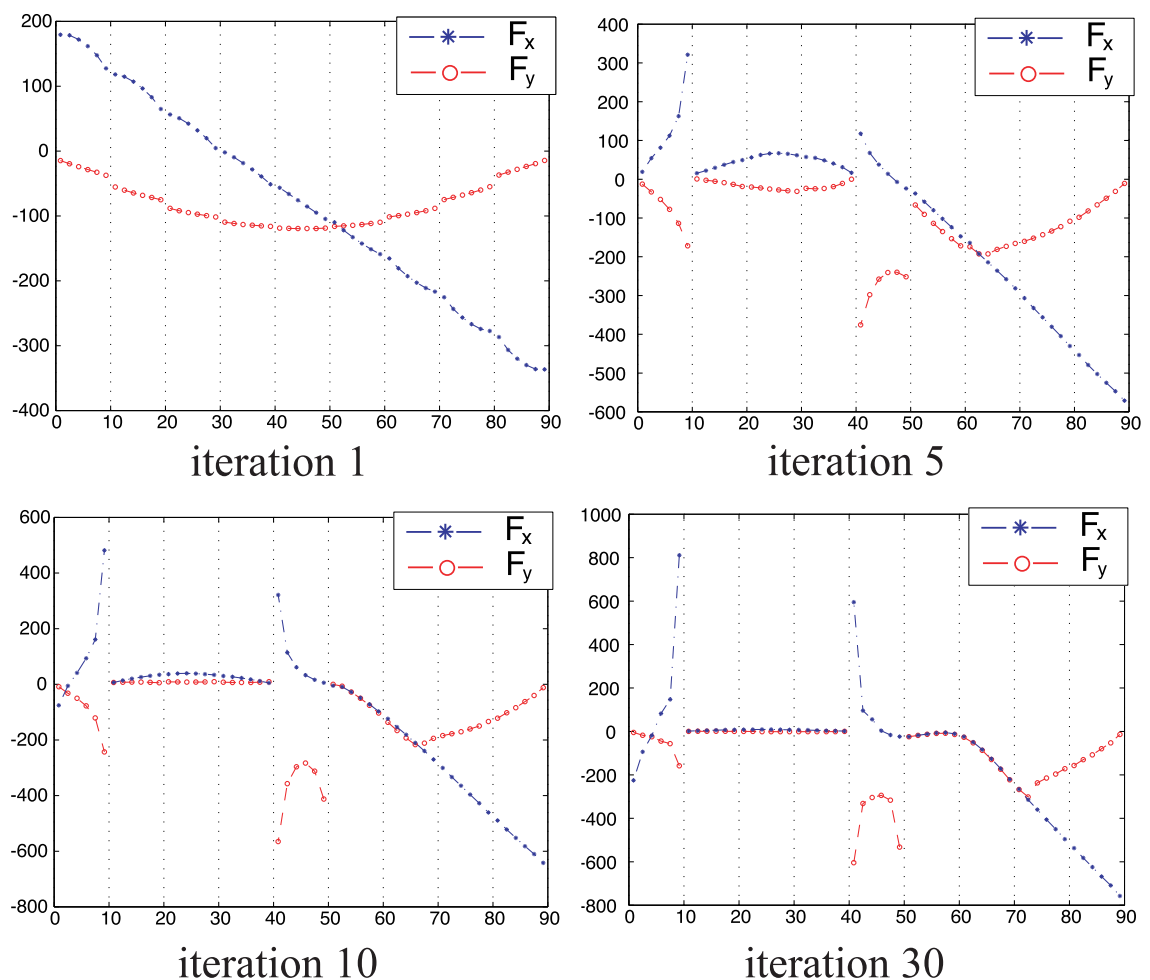

Fig. 13. Contact error criterion on a sliding and sticking crack

\section{Contact criterium on one crack}

normal : Fn. $\Delta W n$

tangential : $(f|F n /-| F t \mid) . / \Delta W t \mid$

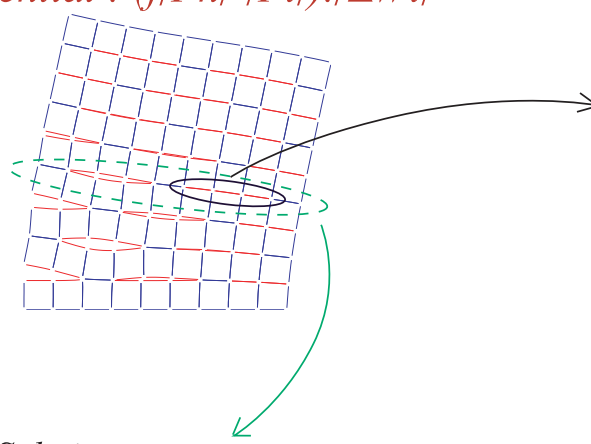

Solution at convergence
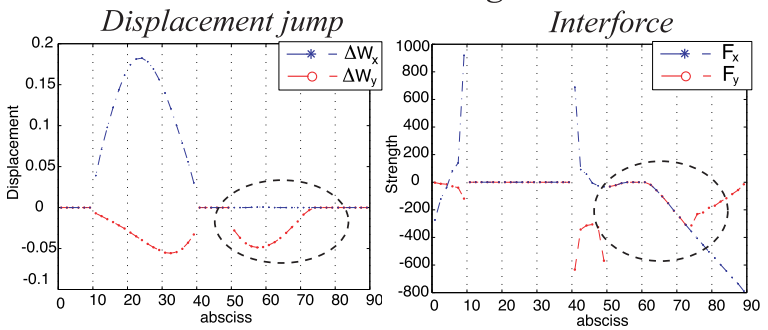

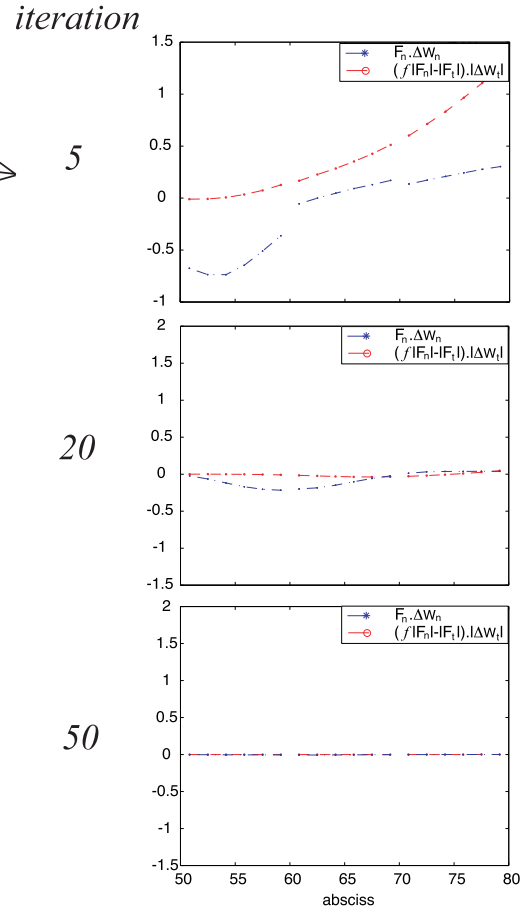

Fig. 14. Contact error criterion on a sliding and sticking crack 


\subsubsection{Scalability of the method}

The evolution of the error is shown on Figure 15 for the problem with $9 \times 9$ subdomains. Now, let us test the convergence rate of the iterative method by

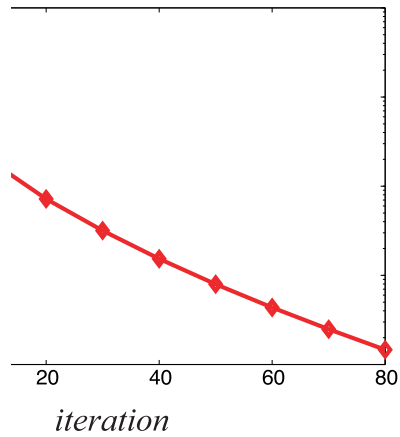

Fig. 15. Evolution of error

analyzing the same problem with different numbers of substructures as shown on Figure 16. We observe that the convergence rate is independent of the number of substructures and conclude that the multiscale method is almost scalable in the case of contact with or without friction.

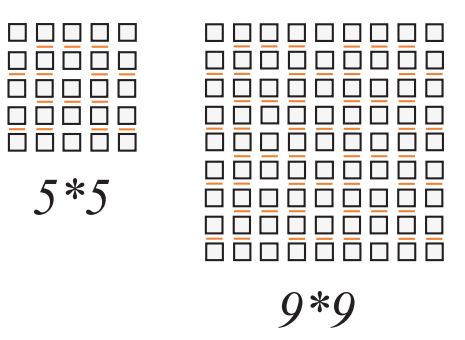

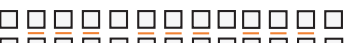

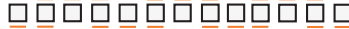

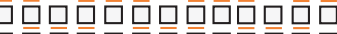

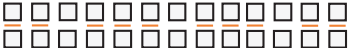

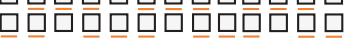

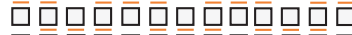

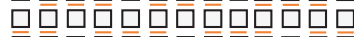

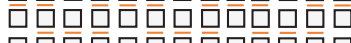

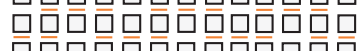

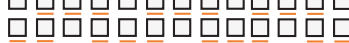

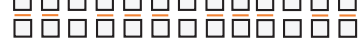

$13 * 13$

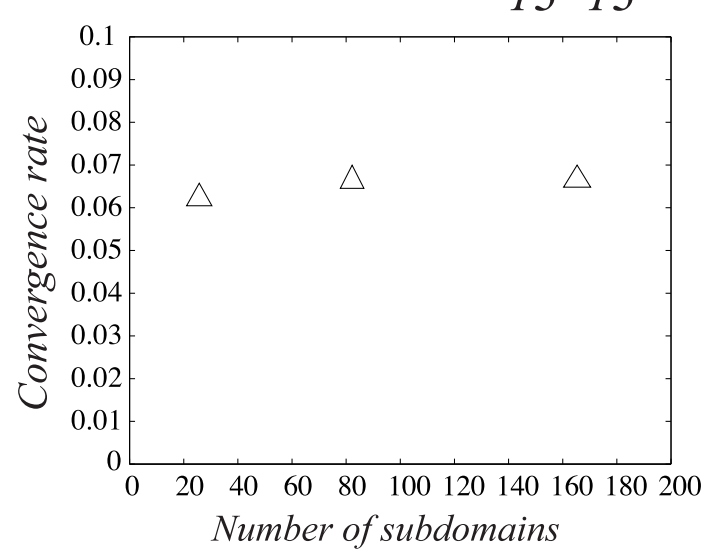

Fig. 16. Scalability test 


\subsubsection{Comparison with other methods}

We compared the efficiency of this method with the previous monoscale method. The new method turned out to be much more efficient than a classical code such as ABAQUS for engineering problems with many contact-type connections; the ratio can exceed 50 (see [5]). The convergence rates of the two methods are compared on Figure 17.

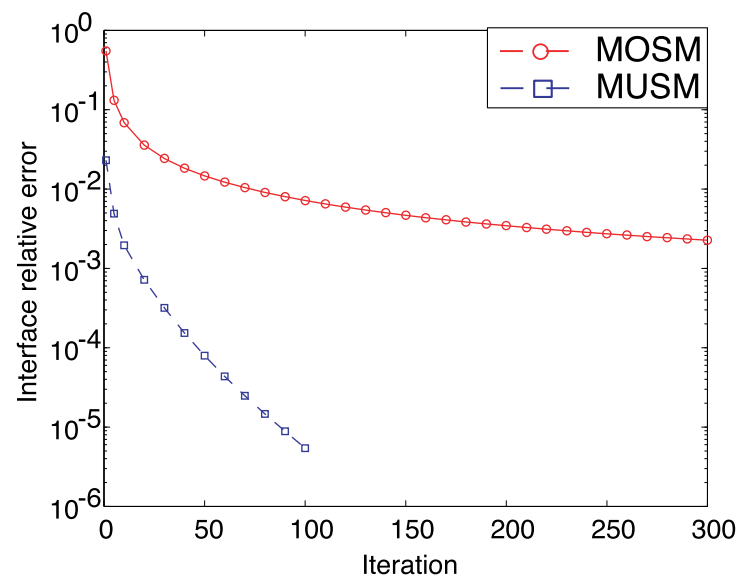

Fig. 17. Comparison between the LATIN monoscale and multiscale methods

We note how efficient MUSM is compared to MOSM. For this comparison, we can look at the stress distribution at iterations 10 and 100 for MUSM (Figure 19) and at iterations 10 and 300 for MOSM (Figure 18). We see that MUSM gives pretty good results at iteration 10 whereas MOSM is still far from having converged at iteration 300 .

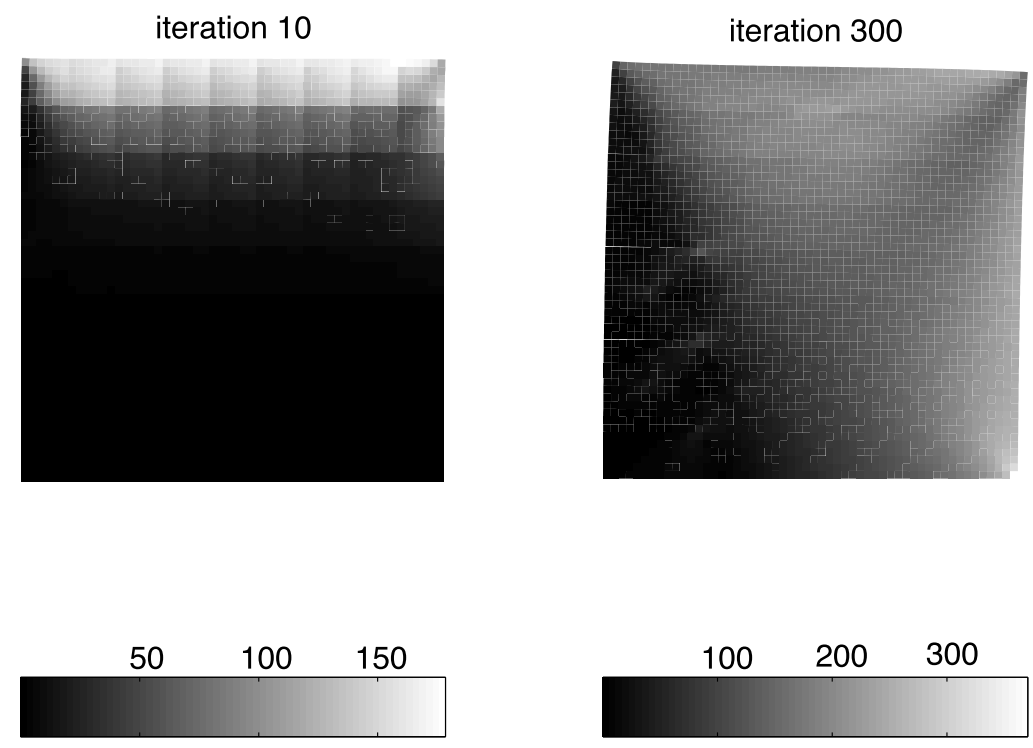

Fig. 18. Von Mises' stress at iterations 10 and 300 for MOSM 

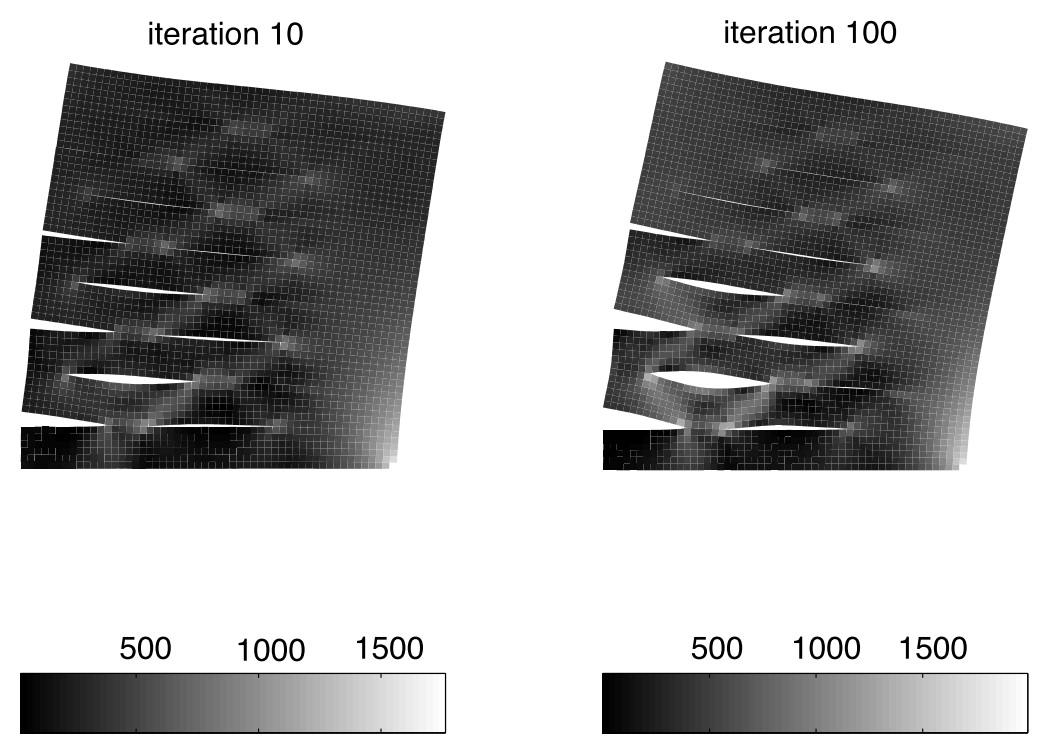

Fig. 19. Von Mises' stress at iterations 10 and 100 for MUSM

Cost of the algorithm

With MUSM, the number of operations to solve the local problem $\boldsymbol{\Gamma}$ is unchanged. For the linear problem $\mathbf{A}_{\mathbf{d}}$, we need approximately twice as many operations as with MOSM. The construction of the homogenized operator $\mathbf{L}_{E}$ consists of solving for each substructure $E \sum_{E^{\prime} \in \mathbf{E}} \operatorname{dim}\left(\mathcal{F}_{\Gamma_{E E^{\prime}}}^{M}\right)$ local problems, where $\operatorname{dim}\left(\mathcal{F}_{\Gamma_{E E^{\prime}}}^{M}\right)$ is the number of macro base functions on Interface $\Gamma_{E E^{\prime}}$. In this example, this is equivalent to solving 16 local problems per substructure. Although we need to build the homogenized operator and perform twice as many operations per iteration of the LATIN method, MUSM remains much more efficient than MOSM.

\section{Conclusion}

The present strategy is a multi-scale approach to solve contact problems. This strategy, which appears to be efficient and robust, leads to a parallel algorithm well-adapted to parallel-architecture computers. The extensibility of the method has been numerically proved for contact problems.

In certain cases, the use of three scales could be interesting from a modeling point of view (e.g. for composites) and/or to improve the computational efficiency. This can be achieved by discretizing the macro problem.

Further work is in progress to extend this computational strategy to timedependent problems. 


\section{References}

[1] P. Alart, A. Curnier, A mixed formulation for frictional contact problems prone to Newton like solution methods, Computer Methods in Applied Mechanics and Engineering, Vol 92, pp 253-275 (1991)

[2] P. Alart, M. Barboteu, P. L. Tallec, M. Vidrascu, Méthode de Schwarz additive avec solveur grossier pour problèmes non symétriques, Comptes Rendus de l'Académie des Sciences, Paris, serie I, pp. 399-404 (2000)

[3] J. S. Arora, A. I. Chahande, J. K. Paeng, Multiplier methods for engineering optimization, International Journal for Numerical Methods in Engineering, 32, pp 1485-1525 (1991)

[4] M. Barboteu, P. Alart, M. Vidrascu, A domain decomposition strategy for nonclassical frictional multi-contact problems, Computer Methods in Applied Mechanics and Engineering, 190, issue 37-38, pp 4785-4803 (2001)

[5] L. Champaney, J.-Y. Cognard, D. Dureisseix, P. Ladevèze , Large scale applications on parallel computers of a mixed domain decomposition method, Computational Mechanics no 19 (1997), pp. 253-263

[6] L. Champaney, J.-Y. Cognard, P. Ladevèze, Modular analysis of assemblages of 3D structures with unilateral contact conditions, Computers and Structures, 73(1/5), pp 249-266 (1999)

[7] G. Dilintas, P. Laurent-Gengoux, D. Trystram, A conjugate projected gradient method with preconditioning for unilateral contact problems, Computers and Structures, Vol 29, no 4, pp 675-680 (1988)

[8] Z. Dostal, A. Friedlander, S. Santos, Solution of coercive and semicoercive contact problems by FETI domain decomposition, Contemporary Mathematics, Vol 218, pp 82-93 (1998)

[9] D. Dureisseix, C. Farhat, A numerical scalable domain decomposition method for the soltuion of frictionless contact problems, Interanational Journal for Numerical Methods in Engineering, 50, pp 2643-2666 (2001)

[10] G. Duvaut, J. L. Lions, Inequalities in Mechanics and Physics, Springer Berlin, (1976)

[11] C. Farhat, F.-X. Roux, A method of finite element tearing and interconnecting and its parallel solution algorithm, Interanational Journal for Numerical Methods in Engineering, 32, pp 1205-1227 (1991)

[12] J. Haslinger, I. Hlavacek, J. Necas, Numerical methods for unilateral problems in solid mechanics, Handbook of Numerical Analysis, ed. by P. G. Ciarlet and J. L. Lions, North Holland (1996)

[13] N. Kikuchi, Penalty/finite element approximations of a class of unilateral contact problems, Penalty Method and Finite Element Method, ASME : New York (1982) 
[14] N. Kikuchi, J. T. Oden (eds), Contact problems in elasticity : A Study of Variational Inequalities and Finite Element Methods, SIAM : Philadelphia (1982)

[15] A. Klarbing, A mathematical programming approach to three-dimensional contact problems with friction, Computer Methods in Applied Mechanics and Engineering, Vol 58, pp 175-200 (1986)

[16] P. Ladevèze, D. Dureisseix, A multi-level and mixed domain decompsition approach for structural analysis, Domain Decomposition Methods, 10, pp 246253. Contemporary Mathematics (1998)

[17] P. Ladevèze, Nonlinear Computational Structural Mechanics - New Approaches and Non-Incremental Methods of Calculation, Springer Verlag, (1999)

[18] P. Ladevèze, D. Dureisseix, A new micro macro computational strategy for structural analysis, Comptes-Rendus de l'Académie des Sciences, Paris, 327, pp 1237-1244 (1999)

[19] P. Ladevèze, D. Dureisseix, A Micro/Macro Approach for Parallel Computing of Heterogeneous Structures, International Journal for Computational Civil and Structural Engineering, Vol 1 (2000), pp. 18-28

[20] P. Ladevèze, O. Loiseau, D. Dureisseix, A micro-macro and parallel computational strategy for highly heterogeneous structures, International Journal for Numerical Methods in Engineering, Vol 52 (2001), pp. 121-138

[21] H.-O. May, The conjugate gradient method for unilateral problems, Computers and Structures, Vol 12, no 4, pp 595-598 (1986)

[22] M. Minoux, Mathematical programming : theory and applications, Wiley (1986)

[23] J. T. Oden, J. A. C. Martins, Models and computational method for dynamic friction phenomena, Computer Methods in Applied Mechanics and Engineering, Vol 52, pp 527-634 (1985)

[24] M. Raous, P. Chabrand, F. Lebon, Numerical methods for frictional contact problems and applications, Journal of Theoretical and Applied Mechanics 7, pp 111-128 (1988)

[25] M. Raous, S. Barbarin, Conjugate gradient for frictional contact, Proceedings of the Contact Mechanics International Symposium, Curnier A. (ed.). Presses Polytechniques et Universitaires Romandes, (1992)

[26] M. Raous, M. Jean, J.-J. Moreau (eds), Proceedings of the Second Contact Mechanics International Symposium, New York, Plenum Press, (1995)

[27] J. C. Simo, T. A. Laursen, An augmented lagrangian treatment of contact problem involving friction, Computers and Structures, Vol 42, pp 97-116 (1992) 
[28] P. Wriggers, Finite element algorithms for contact problems, Archives of Computational Methods in Engineering, 2, pp 1-49 (1995)

[29] P. Wriggers et al., Penalty and augmented Lagrangian formulations for contact problems. In Proceedings of NUMETA Conference, Swansea (1985)

[30] Z. Zhong, J. Mackerle, Static contact problems - a review, Engineering Computations, Vol 9, pp 3-37 (1992) 